\title{
On the binding of nanometric hydrogen-helium clusters in tungsten
}

\author{
G Bonny $^{1}$, P Grigorev $^{1,2,3}$ and D Terentyev ${ }^{1}$ \\ ${ }^{1}$ SCK•CEN, Nuclear Materials Science Institute, Boeretang 200, B-2400 Mol, Belgium \\ 2 FUSION-DC, Department of Applied Physics EA17, Ghent University, Sint-Pietersnieuwstraat 41 B4, \\ B-9000 Gent, Belgium \\ ${ }^{3}$ St. Petersburg State Polytechnical University, Department of Experimental Nuclear Physics K-89, \\ Faculty of Physics and Mechanics, 29 Polytekhnicheskaya str., 195251St. Petersburg, Russia \\ E-mail: gbonny@ sckcen.be
}

Received 3 April 2014, revised 28 August 2014

Accepted for publication 18 September 2014

Published 17 October 2014

\begin{abstract}
In this work we developed an embedded atom method potential for large scale atomistic simulations in the ternary tungsten-hydrogen-helium (W-H-He) system, focusing on applications in the fusion research domain. Following available $a b$ initio data, the potential reproduces key interactions between $\mathrm{H}, \mathrm{He}$ and point defects in $\mathrm{W}$ and utilizes the most recent potential for matrix W. The potential is applied to assess the thermal stability of various $\mathrm{H}-\mathrm{He}$ complexes of sizes too large for $a b$ initio techniques. The results show that the dissociation of $\mathrm{H}-\mathrm{He}$ clusters stabilized by vacancies will occur primarily by emission of hydrogen atoms and then by break-up of $\mathrm{V}-\mathrm{He}$ complexes, indicating that $\mathrm{H}-\mathrm{He}$ interaction does influence the release of hydrogen.
\end{abstract}

Keywords: tungsten, bubbles, interatomic potential

(Some figures may appear in colour only in the online journal)

\section{Introduction}

Tungsten (W) and tungsten based alloys are the primary candidate materials for plasma facing components (PFCs) in fusion reactors. In the DEMO and future commercial reactors PFCs will be exposed to unprecedented and unexplored irradiation conditions. The exposure to high-energy radiation, consisting of neutron damage, helium $(\mathrm{He})$ and hydrogen (H) high temperature/flux plasma, severely damage the microstructure of the materials by violently displacing atoms from their lattice and thereby creating vacancy clusters, dislocation loops, voids and even microscopic bubbles and cracks. All the above mentioned radiation-induced processes cause profound macroscopic property changes that severely degrade the performance and lifespan limits of PFC materials $[1,2]$.

One of the issues in the development of PFCs is the retention of $\mathrm{H}$ isotopes (namely tritium) [3], originating from the trapping of plasma components on pre-existing (i.e. natural) and radiation-induced lattice defects. Without neutron irradiation, experiments involving mixed ion beam and plasma accelerators have shown that under mixed $\mathrm{H}-\mathrm{He}$ exposures the interplay between $\mathrm{H}$ isotopes and $\mathrm{He}$ might have a significant effect on the enhancement of $\mathrm{H}$ retention [4-8]. Moreover, continuous production of displacement damage by neutron scattering will generate lattice defects serving as traps for $\mathrm{H}$ and $\mathrm{He}$, and acting as obstacles to dislocation motion thereby further reducing the ductility of tungsten [2]. Trapping of $\mathrm{H}$ and $\mathrm{He}$ at natural and radiation-induced defects as well as irradiation embrittlement takes its origin at the nano-scale and therefore a good understanding of these phenomena should correspondingly be achieved at the atomic level. This is why the development and application of atomistic tools for W-based systems have recently received essential attention, for example in [9-13].

Theoretical attempts to study the synergy between $\mathrm{H}$ and $\mathrm{He}$ is so far limited to density functional theory (DFT) studies regarding the stability of vacancy-hydrogen-helium $\left(\mathrm{VH}_{l} \mathrm{He}_{m}\right)$ clusters [14-16]. Due to the computational limitations of DFT calculations only elementary clusters containing a single vacancy were considered. However, there is the essential desire to extend our knowledge to the 
stability and mobility of larger $\mathrm{V}_{k} \mathrm{H}_{l} \mathrm{He}_{m}$ clusters (i.e. for $k, l, m \gg 1)$ as their formation certainly occurs under high flux plasma exposure, which typically leads to the formation of well-resolved bubbles and blisters. Also, the study of $\mathrm{H}$ and He accumulation near extended lattice defects, such as dislocation lines, dislocation loops and grain boundaries and their effect on dislocation movement are outside the scope of DFT calculations. Therefore, all such studies require large scale atomistic simulations employing semi-empirical interatomic potentials that are known to offer an acceptable compromise between computational efficiency and physical reliability. For the development of such potentials, DFT data serves as a guide.

In this work we develop an embedded atom method (EAM) potential for the ternary $\mathrm{W}-\mathrm{H}-\mathrm{He}$ system. The potential is benchmarked against available DFT data from the literature and its performance is compared with the only ternary WHHe potential available so far: the bond order potential (BOP) by Li et al [9]. As a first step, we apply the developed potential to extend the information about the thermal stability of $\mathrm{V}_{k} \mathrm{H}_{l} \mathrm{He}_{m}$ clusters beyond the scale accessible to DFT calculations. For convenience and further use in kinetic mean field theory or object kinetic Monte Carlo models, the results are formatted within a frame of a simple yet accurate liquid tear drop (LTD) model.

The paper is organized as follows. In section 2 we explain the fitting strategy followed to fit the potentials and the procedures applied to obtain the binding energy of any $\mathrm{V}_{k} \mathrm{H}_{l} \mathrm{He}_{m}$ cluster. In section 3 we validate the potential by making one-to-one comparisons with both BOP and DFT data. In section 4 we present and discuss the results concerning the stability of $\mathrm{V}_{k} \mathrm{H}_{l} \mathrm{He}_{m}$ clusters. The paper is finalized by conclusions.

\section{Methodology}

In the literature, many EAM type interatomic potentials for bcc $\mathrm{W}$ are available, see e.g. [10-12, 17-30]. A critical review assessing their strengths and weaknesses is given in [13]. For this work, we selected the one, which gives the best 'global performance', i.e. 'EAM2' from the work of Marinica et al [11]. As key features, this potential provides elastic constants, point-defect, edge and screw dislocation properties as well as grain boundary energies consistent with DFT calculations or experiments (see [13] for more details).

In the literature three semi-empirical potentials exist for WHe $[10,31,32]$ and two for WH $[33,34]$. From those potentials, the ones by Wilson et al [31] and Henriksson et al [32] predict opposite stability for $\mathrm{He}$ in an octahedral and tetrahedral position. The one by Juslin and Wirth [10] provides a He migration barrier of $0.21 \mathrm{eV}$, which overestimates the DFT value $(0.06 \mathrm{eV})$ by more than a factor three. The WH potential by Juslin et al [33], on the other hand, predicts the $<110>$ dumbbell self interstitial be more stable than the $<111>$ one in bcc W, which is in contradiction with DFT data (see [13] and references therein). The one by Li et al [34] is part of the ternary WHHe BOP that is used as a benchmark throughout this work. At this point we also note that none of the potentials for pure $\mathrm{W}$ used in the above works reproduce the key features as good as 'EAM2' by Marinica et al [11]. In addition, a 1/2 $<111>$ screw dislocation in bec $\mathrm{W}$ relaxed by BOP provides a threefold degenerate core structure, which is contradictory to DFT results [35] and 'EAM2' by Marinica et al [11].

The $\mathrm{H}-\mathrm{H}$ interaction in bulk $\mathrm{W}$ is essentially different from its description in vacuum. In vacuum, two $\mathrm{H}$ atoms form the strongly bonded $\mathrm{H}_{2}$ molecule $\left(E_{\mathrm{b}}=4.75 \mathrm{eV}\right)$ [33]. In bulk $\mathrm{W}$, on the other hand, two $\mathrm{H}$ atoms exhibit repulsion or weak binding [14] as they cannot form the strongly bonded $\mathrm{H}_{2}$ molecule due to interactions with the surrounding $\mathrm{W}$ atoms. The modulation of such behaviour within the EAM frame work is difficult to achieve. Therefore, we chose to focus on the effective interaction of $\mathrm{H}$ (and $\mathrm{He}$ ) in bulk W. As a consequence, the here derived potentials for $\mathrm{H}$ and $\mathrm{He}$ should not be used in vacuum.

We have fitted two sets of WHHe potentials, namely, EAM1 and EAM2. Both potentials were fitted to reproduce the relative stability between tetrahedral $(\mathrm{T})$ and octahedral $(\mathrm{O})$ sites as well as between tetrahedral and $<110\rangle$ dumbbell position, with the latter serving as saddle for $\mathrm{T}-\mathrm{T}$ migration. In addition, the binding between $\mathrm{H}-\mathrm{H}, \mathrm{He}-\mathrm{He}$ and $\mathrm{H}-\mathrm{He}$ pairs in bulk $\mathrm{W}$ and the binding between vacancy- $\mathrm{H}$ and vacancyHe pairs were fitted. For EAM1, emphasis was put on a quantitative reproduction of DFT data of the binding between $\mathrm{H}-\mathrm{H}, \mathrm{He}-\mathrm{He}$ and $\mathrm{H}-\mathrm{He}$ pairs. The off-centre position of a $\mathrm{H}$ atom in a vacancy as predicted by DFT [36] was not considered, and therefore both $\mathrm{H}$ and $\mathrm{He}$ are described by pair potentials only. For EAM2 we focussed on stabilizing $\mathrm{H}$ in an off-centre position in the vacancy and therefore an embedding function was added for $\mathrm{H}$. For both $\mathrm{H}$ and $\mathrm{He}$ no density function is defined, i.e. only $\mathrm{W}$ adds to the electron density at a given site and there is no contribution to it by $\mathrm{H}$ or He. The optimized parameters for both EAM1 and EAM2 are reported in appendix A.

The total binding energy of a $\mathrm{V}_{k} \mathrm{H}_{l} \mathrm{He}_{m}$ cluster, $E_{\mathrm{b}}^{\text {tot }}$, was calculated as,

$$
\begin{gathered}
E_{\mathrm{b}}^{\mathrm{tot}}\left(V_{k} H_{l} \mathrm{He}_{m}\right)=k E(V)+l E(H)+m E(\mathrm{He}) \\
-E\left(V_{k} H_{l} \mathrm{He}_{m}\right)-(k+l+m-1) E(W)
\end{gathered}
$$

Here $E(\mathrm{~W})$ is the total energy of the perfect bcc W crystal, $E(\mathrm{~V}), E(\mathrm{H})$ and $E(\mathrm{He})$ are the total energy of the bcc W crystal containing one $\mathrm{V}, \mathrm{H}$ and $\mathrm{He}$ atom, respectively, with $\mathrm{H}$ and $\mathrm{He}$ in the tetrahedral position. With this definition, positive values of $E_{\mathrm{b}}^{\text {tot }}$ indicate attraction.

In our study, we have considered $\mathrm{V}_{k} \mathrm{H}_{l} \mathrm{He}_{m}$ clusters containing up to 12 vacancies (in the most compact configuration) with a $\mathrm{H}$ to $\mathrm{V}$ ratio, $x_{\mathrm{H}}$, and $\mathrm{He}$ to $\mathrm{V}$ ratio, $x_{\mathrm{He}}$, in the range 0.1-6. For the selected maximum number of $\mathrm{V}, x_{\mathrm{H}}$ and $x_{\mathrm{He}}$, the sequential binding energy of a $\mathrm{V}, \mathrm{H}$ and $\mathrm{He}$ to a $\mathrm{V}_{k} \mathrm{H}_{l} \mathrm{He}_{m}$ cluster saturates, respectively, so no larger clusters need to be addressed. In total, 2087 different $\mathrm{V}_{k} \mathrm{H}_{l} \mathrm{He}_{m}$ clusters were considered. In each cluster the $\mathrm{H}$ and He were introduced at random (with a maximum of $6 \mathrm{H} / \mathrm{He}$ per vacancy) and the configuration was relaxed ten times using a quench method with a molecular dynamics (MD) run at $300 \mathrm{~K}$ for $1 \mathrm{ps}$ in between each quench down to $0 \mathrm{~K}$. The latter procedure allows the system to evolve out of local minima and 
Table 1. Point defect properties of $\mathrm{H}$ and $\mathrm{He}$ in bcc W calculated by DFT and the potentials.

\begin{tabular}{|c|c|c|c|c|c|c|c|c|}
\hline \multirow[b]{2}{*}{ Property } & \multicolumn{4}{|c|}{ Hydrogen } & \multicolumn{4}{|c|}{ Helium } \\
\hline & DFT & EAM1 & EAM2 & BOP & DFT & EAM1 & EAM2 & BOP \\
\hline$\Delta E$ (Octa-Tetra) $(\mathrm{eV})$ & 0.38 & 0.35 & 0.38 & 0.32 & 0.22 & 0.19 & 0.23 & 0.17 \\
\hline$\Delta E(<100>-$ Tetra $)(\mathrm{eV})$ & 0.39 & 0.35 & 0.38 & 0.32 & 0.23 & 0.19 & 0.23 & 0.17 \\
\hline$\Delta E(<110>$-Tetra $)(\mathrm{eV})$ & 0.21 & 0.22 & 0.21 & 0.22 & 0.07 & 0.09 & 0.06 & 0.02 \\
\hline$\Delta E(<111>-$ Tetra $)(\mathrm{eV})$ & 1.51 & 2.10 & 2.70 & 2.29 & 0.51 & 1.40 & 0.98 & 0.75 \\
\hline$E_{\mathrm{b}}(\mathrm{X}-\mathrm{Vac})(\mathrm{eV})$ & 1.19 & 1.24 & 1.33 & 2.03 & 4.55 & 4.55 & 4.54 & 5.04 \\
\hline$E_{\mathrm{m}}(\mathrm{X})(\mathrm{ev})$ & 0.20 & 0.22 & 0.21 & 0.22 & 0.06 & 0.09 & 0.06 & 0.02 \\
\hline
\end{tabular}

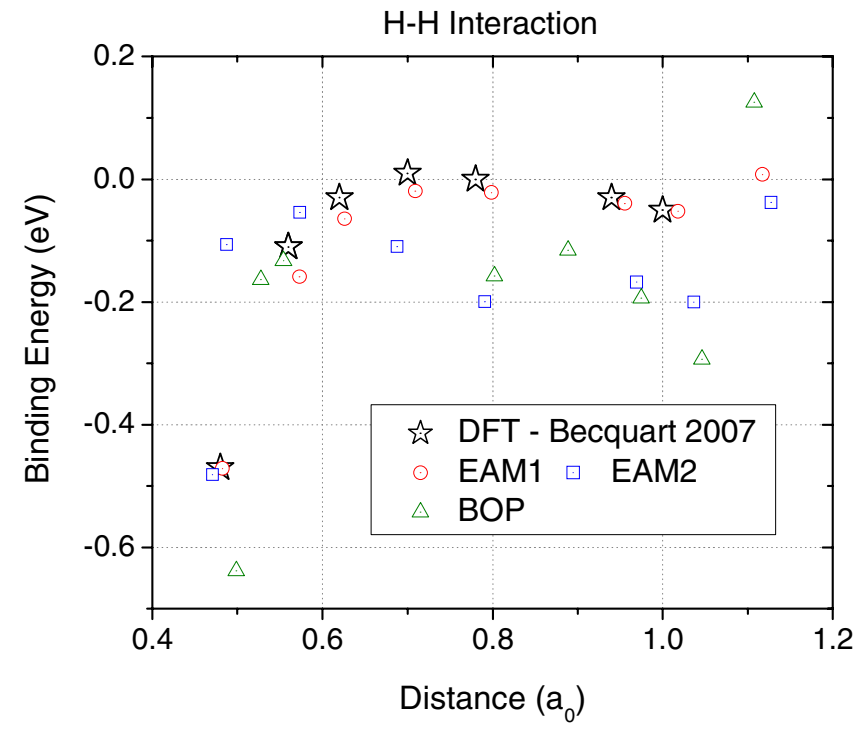

Figure 1. Comparison of the $\mathrm{H}-\mathrm{H}$ binding energy as a function of distance as calculated by DFT and the potentials.

was tested to provide an absolute minimum in the case of the single vacancy. For each configuration, the lowest of the 10 values for the total energy was retained. For all runs, a cubic bcc $\mathrm{W}$ crystal was used containing 2000 atoms with periodic boundaries in all directions.

\section{Validation of the potentials}

In table 1 the point defect properties of $\mathrm{H}$ and $\mathrm{He}$ in bcc $\mathrm{W}$ calculated with our potentials are compared to DFT [14] and BOP [9]. Clearly, DFT predicts the tetrahedral position to be the most favourable for both $\mathrm{H}$ and $\mathrm{He}$, which is reproduced by all potentials. In addition, all potentials predict the correct ordering in interstitial formation energies, although EAM1 and EAM2 show the best quantitative agreement with DFT. With respect to the binding energy between a $\mathrm{H}$ or $\mathrm{He}$ to a vacancy, both EAM1 and EAM2 closely reproduce the DFT values, while BOP underestimates and overestimates the binding for $\mathrm{H}$ and $\mathrm{He}$, respectively. The migration energy for $\mathrm{H}$ is well reproduced by all potentials, but the one for $\mathrm{He}$ is only reproduced by EAM1 and EAM2, and underestimated by BOP by a factor three.

In figures $1-3$ the binding energy between $\mathrm{H}-\mathrm{H}, \mathrm{He}-\mathrm{He}$ and $\mathrm{H}-\mathrm{He}$ pairs as a function of separation distance is plotted as calculated by DFT and the potentials. The

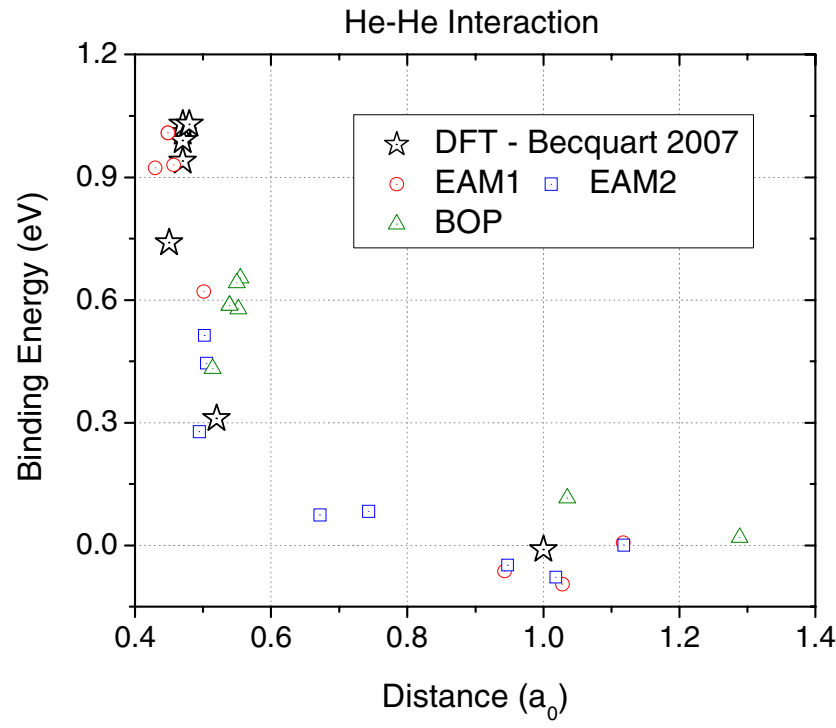

Figure 2. Comparison of the He-He binding energy as a function of distance as calculated by DFT and the potentials.

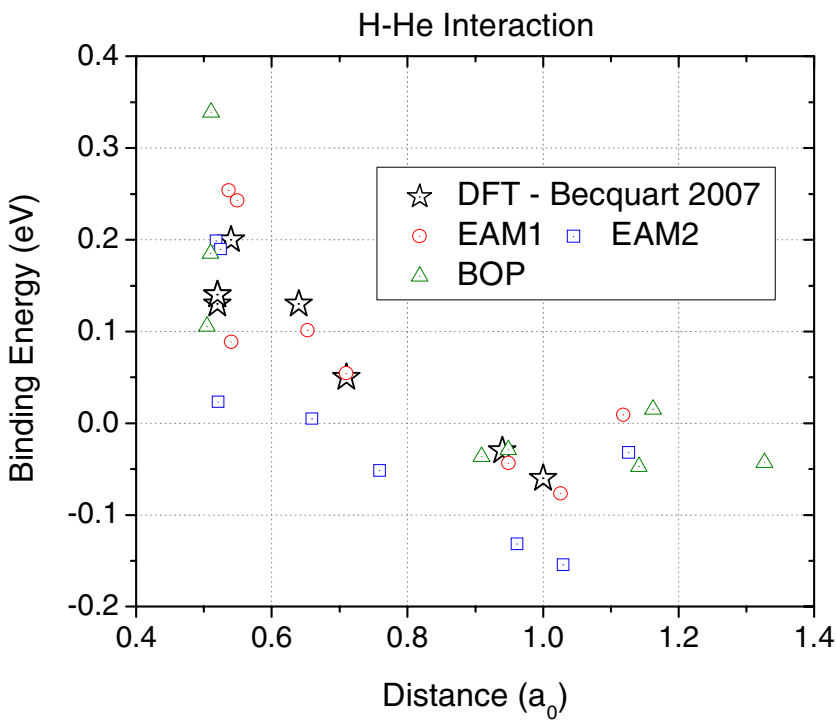

Figure 3. Comparison of the $\mathrm{H}-\mathrm{He}$ binding energy as a function of distance as calculated by DFT and the potentials.

considered configurations are taken from [14] and only the final distance between the pairs is considered. As with both DFT and the potentials the distance between $\mathrm{H}$ and He pairs changes considerably during atomic relaxation, a comparison of binding energy with distance is more sensible than a 


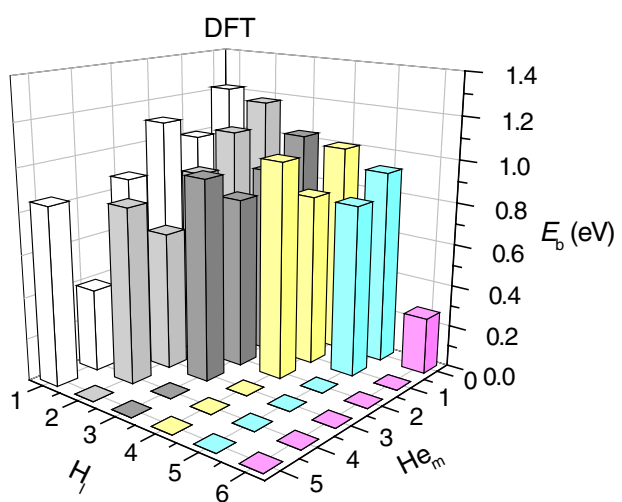

(a)

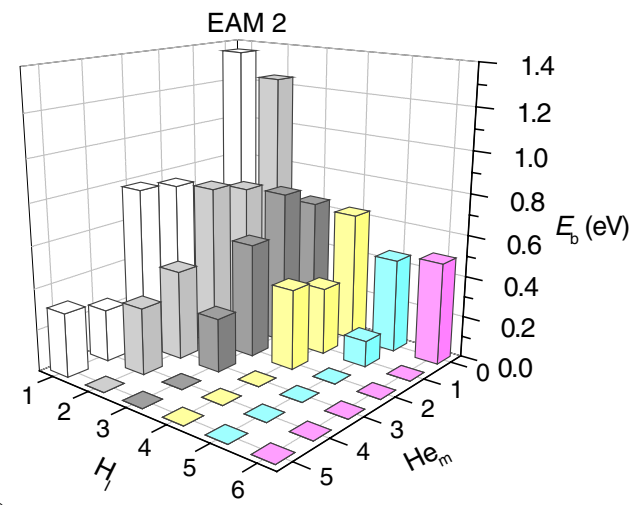

(c)

Figure 4. Binding energy of a $\mathrm{H}$ in a $\mathrm{VH}_{l} \mathrm{He}_{m}$ cluster as calculated by (a) DFT, (b) EAM1, (c) EAM2 and (d) BOP. one-to-one comparison between the specific configurations. Taking DFT as the reference, $\mathrm{He}-\mathrm{He}$ and $\mathrm{He}-\mathrm{H}$ attract with maximum values of $1.03 \mathrm{eV}$ and $0.20 \mathrm{eV}$, respectively, while $\mathrm{H}-\mathrm{H}$ pairs repel with a maximum of $-0.47 \mathrm{eV}$. This behaviour is qualitatively reproduced by all potentials and also quantitatively by EAM1. Both BOP and EAM2 have a too long interaction range for $\mathrm{H}-\mathrm{H}$ pairs, underestimate the $\mathrm{He}-$ $\mathrm{He}$ attraction and overestimate (BOP) the $\mathrm{H}-\mathrm{He}$ binding or underestimate (EAM2) the $\mathrm{H}-\mathrm{He}$ interaction range.

In figures 4 and 5 the binding energy of a $\mathrm{H}$ and $\mathrm{He}$ atom to $\mathrm{VH}_{l} \mathrm{He}_{m}$ clusters calculated by the potentials and DFT [14] is compared. The DFT results show that He is bound stronger than $\mathrm{H}$ to the same $\mathrm{VH}_{l} \mathrm{He}_{m}$ cluster by roughly a factor four. For both $\mathrm{H}$ and $\mathrm{He}$, the binding energy slightly decreases with increasing cluster size. For EAM1, the values for the binding energy are within the DFT range, but with increasing He content, the binding of He to the cluster does not decrease. For EAM2, the values for the binding energy are also within the DFT range, and qualitatively, also the binding energy decreases with cluster size. For BOP, the binding energy for $\mathrm{H}$ is overestimated by about a factor two, but for $\mathrm{He}$, on the other hand, the values lay within the DFT range. Qualitatively, BOP reproduces the decrease in binding energy with cluster size.

\section{Binding energy of $\mathrm{VH}_{l} \mathrm{He}_{m}$ clusters}

From the results presented in section 3 with respect to $\mathrm{VH}_{l} \mathrm{He}_{m}$ clusters, EAM2 seems the most suitable to extend the calculations to $\mathrm{VH}_{l} \mathrm{He}_{m}$ clusters. In figure 6, the data as calculated by molecular dynamics (MD) is presented as scatter for clusters containing 2, 4 and 6 vacancies. For both interand extra-polation purposes, we have fitted a 3D hyper surface based on a LTD model to represent the data, as detailed in appendix B. From this function, all sequential binding energies can easily be derived. Projections for clusters containing 2, 4 and 6 vacancies are superposed in figure 6 . The average deviation between raw data and fitted surface is $\sim 10 \%$, which is acceptable for a simple LTD model.

As an additional validation of the model, we show the binding energy of a $\mathrm{He}$ atom to a $\mathrm{VHe}_{m}$ cluster (figure 7(a)) and of a $\mathrm{H}$ atom to a $\mathrm{VH}_{l}$ and $\mathrm{VHeH}_{l}$ cluster (figure 7(b)) computed with the LTD model, DFT and EAM2. For $\mathrm{VHe}_{m}$, the LTD model slightly overestimates the DFT and EAM2 results, but agreement is nevertheless satisfactory. For $\mathrm{VHeH}_{l}$ and $\mathrm{VH}_{l}$, the difference between the DFT data sets (and EAM2 data sets) is negligible. This trend is followed by the LTD model, although the values are somewhat underestimated. Considering all approximations, we find the LTD model satisfactory and this enables to extrapolate to larger $\mathrm{V}_{k} \mathrm{H}_{l} \mathrm{He}_{m}$ clusters.

In figure 8 we present the dissociation energy of a $\mathrm{V}, \mathrm{H}$ and $\mathrm{He}$ in a VHHe cluster as a function of $x_{\mathrm{H}}$ and $x_{\mathrm{He}}$ for the limiting cases of clusters containing two (figure 8(a)) and 12 (figure $8(b)$ ) vacancies. The dissociation energy was obtained in the standard way as the sum of the sequential binding energy of a $\mathrm{V} / \mathrm{H} / \mathrm{He}$ atom to the cluster and its migration energy. For a vacancy the sequential binding energy is obtained as, 


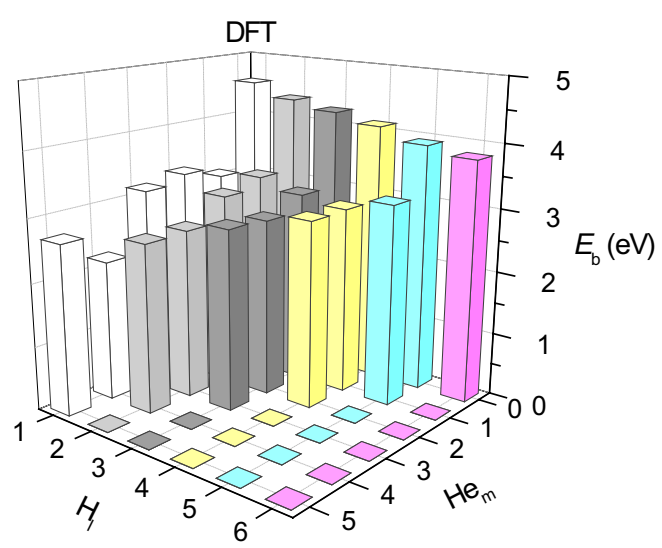

(a)

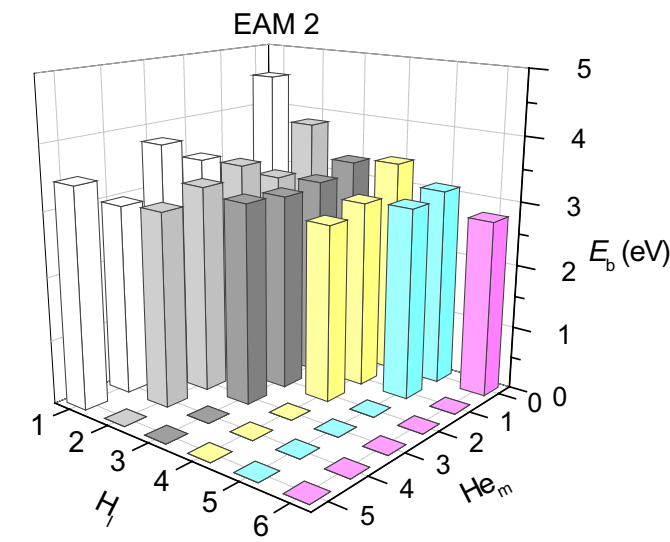

(c)

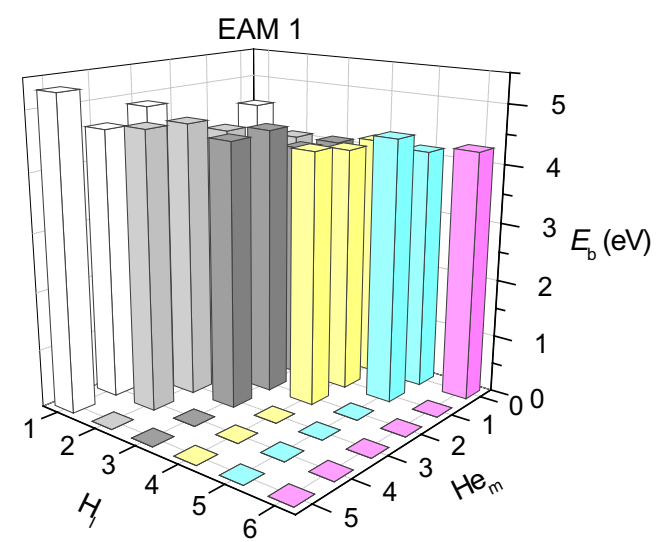

(b)

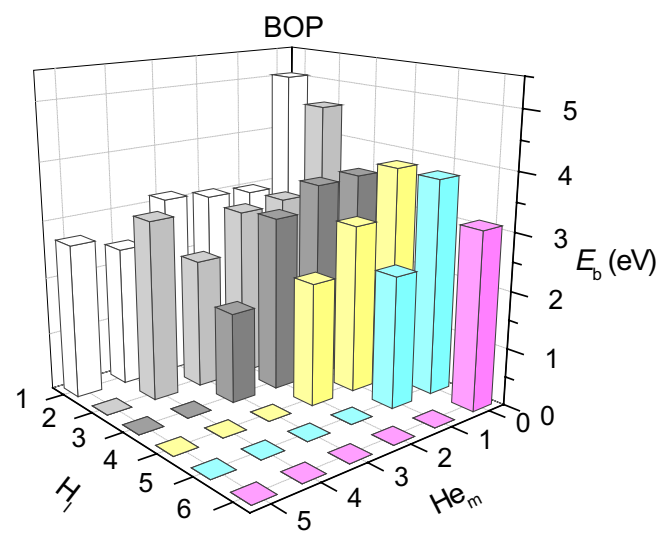

(d)

Figure 5. Binding energy of a $\mathrm{He}$ in a $\mathrm{VH}_{l} \mathrm{He}_{m}$ cluster as calculated by (a) DFT, (b) EAM1, (c) EAM2 and (d) BOP

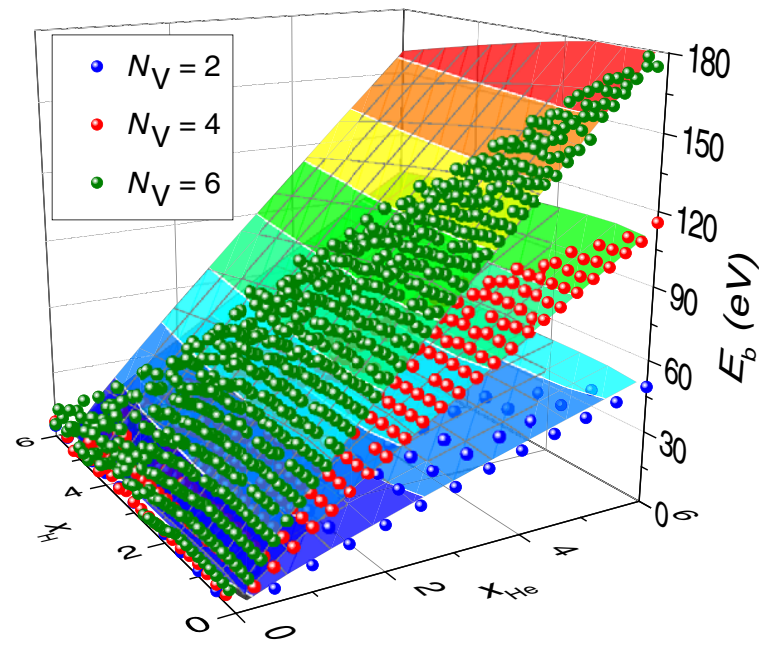

Figure 6. Total binding energy of VHHe clusters as a function of hydrogen- and helium-vacancy ratio $\left(x_{\mathrm{H}}, x_{\mathrm{He}}\right)$ for clusters containing two, four and six vacancies.

$E_{\mathrm{b}}^{\text {tot }}\left(V_{k} H_{l} \mathrm{He}_{m}\right)-E_{\mathrm{b}}^{\text {tot }}\left(V_{k-1} H_{l} \mathrm{He}_{m}\right)$, with similar expressions for $\mathrm{H}$ and $\mathrm{He}$. As values for the migration energy we used the ones provided by the potential $\left(E_{\mathrm{m}}(V)=1.85 \mathrm{eV}\right)$ [13], which is within the range of experiments and DFT calculations [13].

We observe that for both limiting cases the binding energy of $\mathrm{H}$ is lower than that of a V or He for all $x_{\mathrm{H}}$ and $x_{\mathrm{He}}$ combinations, consistent with the single vacancy case (see figures 4 and 5). This implies that any VHHe cluster formed in $\mathrm{W}$ will first release all $\mathrm{H}$ before releasing a $\mathrm{He}$ or $\mathrm{V}$. For VHe clusters, an optimum $x_{\mathrm{He}}$ exists above which He dissociates from the clusters and below which a $\mathrm{V}$ dissociates from the cluster. This optimum, indicated by the dashed line in figures $8(a)$ and $(b)$, depends slightly on $x_{\mathrm{H}}$ and the number of vacancies in the clusters. The optimum point slightly increases with increasing $x_{\mathrm{H}}$ and saturates from $\sim 2$ for two vacancies to $\sim 1$ for six vacancies and more. In addition, the sequential binding of $\mathrm{H}$ to a cluster decreases with increasing number of vacancies while the sequential binding of He to the cluster increases with the number of vacancies. For the sequential binding energy of a vacancy the slope of the surface gets steeper with increasing number of vacancies.

\section{Conclusions}

We have developed two versions of a EAM potential for large scale atomistic simulations in the ternary $\mathrm{W}-\mathrm{H}-\mathrm{He}$ system. Both potentials reproduce key interactions between $\mathrm{H}, \mathrm{He}$ and point defects calculated by DFT. We applied the potentials to compute the dissociation energy of various VHHe clusters of nano-metric size and parameterize a simple liquid-tear drop model applicable to up-scale mean field or kinetic Monte Carlo simulations. The obtained results show that the dissociation of mixed VHHe clusters primarily takes place by emission 


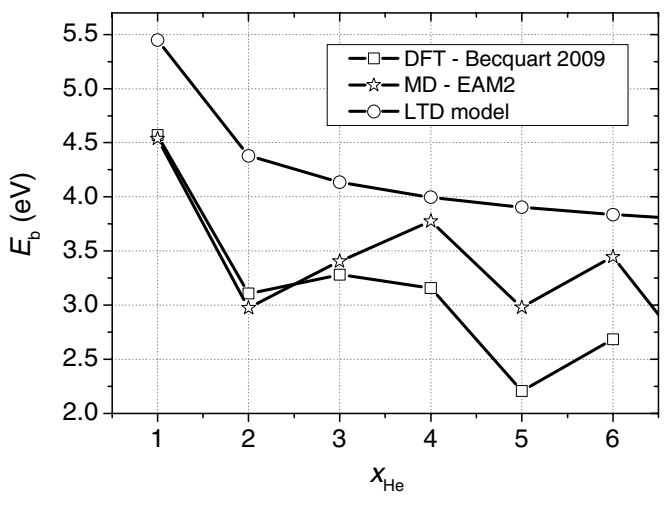

(a)

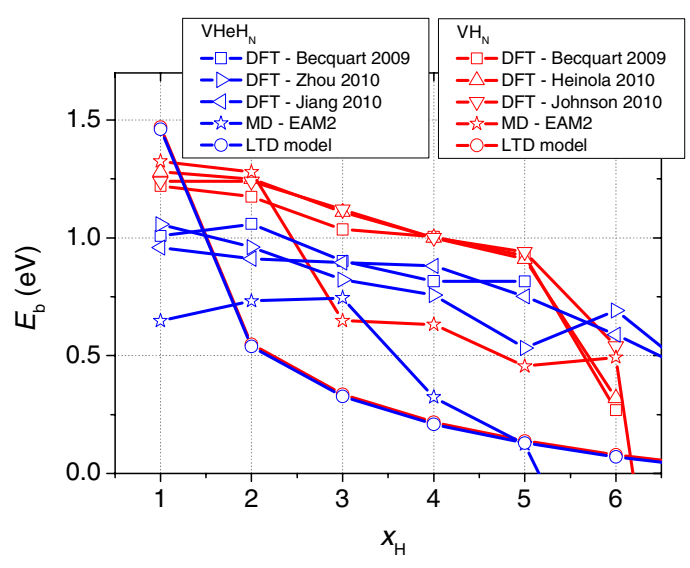

(b)

Figure 7. Binding energy of $(a) \mathrm{He}$ and $(b) \mathrm{H}$ to $(a) \mathrm{VHe}$ and $(b) \mathrm{VHeH}$ clusters, respectively.

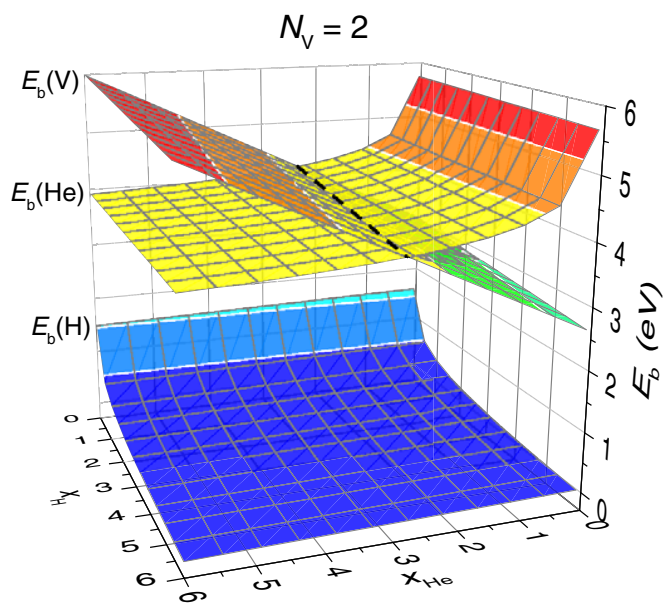

(a)

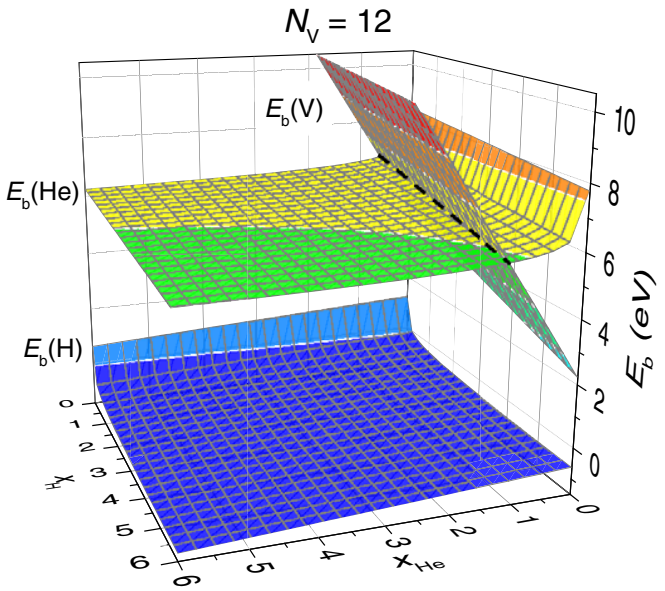

(b)

Figure 8. Dissociation energy for a $\mathrm{V}, \mathrm{H}$ and $\mathrm{He}$ from VHHe clusters as function of hydrogen- and helium ratio $\left(x_{\mathrm{H}}, x_{\mathrm{He}}\right)$ for a cluster containing $(a)$ two and $(b)$ twelve vacancies.

of $\mathrm{H}$, whose trapping energy is not essentially changed by the presence $\mathrm{He}$ in the clusters. Hence, the $\mathrm{H}-\mathrm{He}$ interaction does not affect the thermal stability of $\mathrm{H}$ in the vacancy-stabilized $\mathrm{H}-\mathrm{He}$ clusters. Therefore we conclude that the origin of the $\mathrm{H}-\mathrm{He}$ synergy expressed by the enhanced $\mathrm{H}$ uptake should be investigated at the stage of the nucleation of $\mathrm{H}-\mathrm{He}$ defects.

\section{Acknowledgments}

This work, supported by the European Commission under the Contract of Association between EURATOM/SCK•CEN, was carried out within the framework of the European Fusion Development Agreement. We thank Professor C S Becquart for providing us with raw data of her DFT calculations. We also thank Professor R C Pasianot for his critical remarks during the preparation of this paper.

\section{Appendix A. Parameterization interatomic potential}

The atomic interactions are described using the EAM [37]. In addition to pair interactions, $V$, this approach includes an embedding energy, $F$, dependent on the local electron density, $\rho$. The latter term approximates the many-body contribution of all nearby atoms. The total energy within EAM is given as,

$$
E=\frac{1}{2} \sum_{\substack{i, j=1 \\ j \neq i}}^{N} V_{t_{i} t_{j}}\left(r_{i j}\right)+\sum_{i=1}^{N} F_{t_{i}}\left(\rho_{i}\right) .
$$

Here $N$ represents the total number of atoms in the system, $r_{i j}$ is the distance between atoms $i$ and $j$, and $t_{i}$ denotes chemical species. The local electron density around atom $i$,

Table A1. Spline coefficients for the modified embedding function, $F^{\text {mod }}$.

\begin{tabular}{l}
\hline Spline coefficient \\
\hline$A_{0}=-5.524855802 \mathrm{E}+00$ \\
$A_{1}=2.317313103 \mathrm{E}-01$ \\
$A_{2}=-3.665345949 \mathrm{E}-02$ \\
$A_{3}=8.989367404 \mathrm{E}-03$ \\
\hline
\end{tabular}


Table A2. The optimized fitting parameters for the $\mathrm{WHeH}$ potentials.

\begin{tabular}{|c|c|c|c|c|}
\hline \multirow[b]{2}{*}{$k$} & \multicolumn{2}{|c|}{ EAM1 } & \multicolumn{2}{|c|}{ EAM2 } \\
\hline & $r_{k}(\AA)$ & $a_{k}\left(\mathrm{eV} \AA^{-3}\right)$ & $r_{k}(\AA)$ & $a_{k}\left(\mathrm{eV} \AA^{-3}\right)$ \\
\hline \multicolumn{5}{|c|}{$V_{\mathrm{HeHe}}$} \\
\hline 1 & $2.000000000 \mathrm{E}+00$ & $2.106615791 E+00$ & $1.800000000 \mathrm{E}+00$ & $2.000000000 \mathrm{E}+01$ \\
\hline 2 & $3.000000000 \mathrm{E}+00$ & $-2.217639348 E-01$ & $2.000000000 \mathrm{E}+00$ & $1.051327582 \mathrm{E}+00$ \\
\hline 3 & & & $2.100000000 \mathrm{E}+00$ & $-4.000000000 \mathrm{E}+00$ \\
\hline 4 & & & $3.000000000 \mathrm{E}+00$ & $3.203322671 \mathrm{E}-02$ \\
\hline \multicolumn{5}{|c|}{$V_{\mathrm{HH}}$} \\
\hline 1 & $2.000000000 \mathrm{E}+00$ & $4.862785907 \mathrm{E}-01$ & $1.600000000 \mathrm{E}+00$ & $4.000000000 \mathrm{E}+01$ \\
\hline 2 & $3.000000000 \mathrm{E}+00$ & $1.018797872 \mathrm{E}-01$ & $2.000000000 \mathrm{E}+00$ & $2.315124670 \mathrm{E}-01$ \\
\hline 3 & & & $2.200000000 \mathrm{E}+00$ & $-2.000000000 \mathrm{E}-01$ \\
\hline 4 & & & $3.000000000 \mathrm{E}+00$ & $5.180584543 \mathrm{E}-02$ \\
\hline \multicolumn{5}{|c|}{$V_{\mathrm{HHe}}$} \\
\hline 1 & $1.800000000 \mathrm{E}+00$ & $1.500000000 \mathrm{E}+01$ & $2.000000000 \mathrm{E}+00$ & $3.256370012 \mathrm{E}+00$ \\
\hline 2 & $2.000000000 \mathrm{E}+00$ & $2.563700119 \mathrm{E}-01$ & $2.500000000 \mathrm{E}+00$ & $-4.000000000 \mathrm{E}-01$ \\
\hline 3 & $3.000000000 \mathrm{E}+00$ & $-4.489510592 \mathrm{E}-02$ & $3.000000000 \mathrm{E}+00$ & $-4.489510592 \mathrm{E}-02$ \\
\hline \multicolumn{5}{|c|}{$V_{\mathrm{WHe}}$} \\
\hline 1 & $1.900000000 \mathrm{E}+00$ & $2.100000000 \mathrm{E}+01$ & $1.900000000 \mathrm{E}+00$ & $0.000000000 \mathrm{E}+00$ \\
\hline 2 & $2.200000000 \mathrm{E}+00$ & $8.565323293 \mathrm{E}-01$ & $2.000000000 \mathrm{E}+00$ & $1.400000000 \mathrm{E}+01$ \\
\hline 3 & $3.500000000 \mathrm{E}+00$ & $2.750099819 \mathrm{E}-01$ & $2.200000000 \mathrm{E}+00$ & $-3.712116187 \mathrm{E}+00$ \\
\hline 4 & & & $3.500000000 \mathrm{E}+00$ & $3.105031456 \mathrm{E}-01$ \\
\hline \multicolumn{5}{|c|}{$V_{\mathrm{WH}}$} \\
\hline 1 & $2.000000000 \mathrm{E}+00$ & $1.375733214 \mathrm{E}+01$ & $2.000000000 \mathrm{E}+00$ & $4.424459079 \mathrm{E}+01$ \\
\hline 2 & $3.000000000 \mathrm{E}+00$ & $1.296071475 \mathrm{E}-01$ & $2.647500000 \mathrm{E}+00$ & $-4.993477782 E+00$ \\
\hline 3 & & & $3.295000000 \mathrm{E}+00$ & $1.461712984 \mathrm{E}+00$ \\
\hline
\end{tabular}

contributed from its neighbours is given as,

$$
\rho_{i}=\sum_{\substack{j=1 \\ j \neq i}}^{N} \varphi_{t_{j}}\left(r_{i j}\right)
$$

where $\varphi$ denotes the electron density function of the considered element.

For pure W, 'EAM2' developed by Marinica et al [11] was used. However, we apply some minor modifications that do not modify the potential's properties but exclude possible future problems when $\mathrm{W}$ is alloyed (with e.g. Ta, $\mathrm{Re}, \ldots$ ). Firstly, we transform the potential into its effective gauge $[17,38]$, characterized by an equilibrium density $\rho_{0}=1$ and $F^{\text {eff' }^{\prime}}(1)=0$. The gauge transformation is given as,

$$
\left\{\begin{array}{c}
V^{\mathrm{eff}}(r)=V(r)-2 C \varphi(r) \\
\varphi^{\mathrm{eff}}(r)=S \varphi(r) \\
F^{\mathrm{eff}}(\rho)=F\left(\frac{\rho}{S}\right)+\frac{C}{S} \rho
\end{array},\right.
$$

with $C=1.848055990 \mathrm{E}+00$ and $S=2.232322602 \mathrm{E}-01$. After this transformation, the embedding function, $F^{\text {eff }}$, was modified beyond the inflection point to provide a positive curvature for all densities. The modified embedding function, $F^{\text {mod }}$, is then defined as,

$F^{\mathrm{mod}}(\rho)=\left\{\begin{array}{c}F^{\mathrm{eff}}(\rho), \rho \leqslant \rho_{i} \\ A_{0}+A_{1} \rho+A_{2} \rho^{2}+A_{3} \rho^{3}, \rho>\rho_{i}\end{array}\right.$,

with $\rho_{i}=1.359141225 \mathrm{E}+00$ the inflexion point and $\left\{A_{i}\right\}$ spline coefficients (see table A1) fitted to be continuous at $\rho_{i}$ up to second derivative with $F^{\text {eff }}$. This modification does not change the equilibrium properties of the potential, such as, elastic constants, lattice stabilities, formation energy of point defects and dislocation core structure.

The pair potentials fitted here are parameterized by the cubic spline expansion,

$$
\mathrm{V}(r)=\sum_{k=1}^{N} a_{k}\left(r_{k}-r\right)^{3} H\left(r_{k}-r\right),
$$

where $N$ denotes the total number of knots, $r_{k}$ the knots, $a_{k}$ the fitting parameters and $H$ the Heaviside unit step function. The embedding function for H (EAM2) is parameterized as,

$$
F(\rho)=A \sqrt{\rho}+B \rho^{2},
$$

with $A=-2.610066441 \mathrm{E}+01$ and $B=4.688963869 \mathrm{E}-$ 01 . The optimized knots and fitting parameters for the pair potentials are given in table A2. We note that for both $\mathrm{H}$ and $\mathrm{He}$ no density function is defined, i.e. only $\mathrm{W}$ adds to the electron density at a given site and there is no contribution to it by $\mathrm{H}$ or $\mathrm{He}$.

\section{Appendix B. Parameterization of the total binding energy of $\mathrm{V}_{k} \mathrm{H}_{l} \mathrm{He}_{m}$ clusters}

The total binding energy of $\mathrm{V}_{k} \mathrm{H}_{l} \mathrm{He}_{m}$ clusters was parameterized by a mixture of a LTD model and RedlichKister (RK) expansion. The LTD model consists of two terms: one proportional with volume, expressing the energy gain of the defects clustering together; and one proportional with interface area, expressing the energy loss due to the interface created between matrix and defect cluster. A RK expansion is 
Table B1. Optimized parameters for the LTD model.

\begin{tabular}{lll}
\hline LTD model parameters & & \\
\hline$A_{\mathrm{V}}=4.524241 \mathrm{E}+00$ & $B_{\mathrm{V}}=5.985280 \mathrm{E}+00$ & $L_{\mathrm{VH}}^{0}=-3.727769 \mathrm{E}-02$ \\
$A_{\mathrm{H}}=-7.277276 \mathrm{E}-01$ & $B_{\mathrm{H}}=-2.235115 \mathrm{E}+00$ & $L_{\mathrm{VHe}}^{0}=2.659537 \mathrm{E}-01$ \\
$A_{\mathrm{He}}=2.591062 \mathrm{E}+00$ & $B_{\mathrm{He}}=-2.592230 \mathrm{E}+00$ & $L_{\mathrm{HHe}}^{0}=-9.119871 \mathrm{E}-03$ \\
\hline
\end{tabular}

commonly used to parameterize free energy surfaces of solid solutions in thermodynamic modelling. It accounts for the interactions between the different defect types. In our model we included binary interactions only as the inclusion of ternary interactions did not improve the fit. Applying both models, the total binding energy of a $\mathrm{V}_{k} \mathrm{H}_{l} \mathrm{He}_{m}$ clusters is given as,

$E_{\mathrm{b}}^{\mathrm{tot}}\left(V_{k} H_{l} \mathrm{He}_{m}\right)=A_{\mathrm{V}} k-B_{\mathrm{V}} k^{2 / 3}+A_{\mathrm{H}} l-B_{\mathrm{H}} l^{2 / 3}+A_{\mathrm{He}} m$ $-B_{\mathrm{He}} m^{2 / 3}+L_{\mathrm{VH}}^{0} k l+L_{\mathrm{VHe}}^{0} k m+L_{\mathrm{HHe}}^{0} l m$.

The optimized parameters for this expression are given in table B1.

\section{References}

[1] Zinkle S J 2005 Phys. Plasmas 12058101

[2] Pintsuk G 2012 Comprehensive Nucl. Mater. 4551

[3] Roth J et al 2008 Plasma Phys. Control. Fusion 50103001

[4] Hino T, Koyama K, Yamaguchi Y and Hirohata Y 1998 Fusion Eng. Des. 39-40 227

[5] Nagata S and Takahiro K 2001 J. Nucl. Mater. 290-293 135

[6] Iwakiri H, Morishita K and Yoshita N 2002 J. Nucl. Mater. 307-311 135

[7] Lee H T, Haasz A A, Dawis J W and Macaulay-Newcombe R G 2007 J. Nucl. Mater. 360196

[8] Lee H T, Haasz A A, Davis J W, Macaulay-Newcombe R G, Whyte D G and Wright G M 2007 J. Nucl. Mater. 363-365 898

[9] Li X-C, Shu X, Liu Y-N, Yu Y, Gao F and Lu G-H 2012 J. Nucl. Mater. 42631

[10] Juslin N and Wirth B D 2013 J. Nucl. Mater. 43261

[11] Marinica M-C et al 2013 J. Phys.: Condens. Matter. 25395502

[12] Wang J, Zhou Y L, Li M and Hou Q 2014 Modelling Simul. Mater. Sci. Eng. 22015004

[13] Bonny G, Terentyev D, Bakaev A, Grigorev P and Van Neck D 2014 Modelling Simul. Mater. Sci. Eng. 22053001

[14] Becquart C S and Domain C 2009 J. Nucl. Mater. 386-388 109

[15] Jiang B, Wan F R and Geng W T 2010 Phys. Rev. B 81134112
[16] Zhou H-B, Liu Y-L, Jin S, Zhang Y, Luo G-N and Lu G-H 2010 Nucl. Fusion 50115010

[17] Finnis M W and Sinclair J E 1984 Phil. Mag. A 5045

[18] Ackland G J and Thetford R 1987 Phil. Mag. A 5615

[19] Johnson R A and Oh D J 1989 J. Mater. Res. 41195

[20] Foiles S M 1993 Phys. Rev. B 484287

[21] Wang Y R and Boercker D B 1995 J. Appl. Phys. 78122

[22] Zhou X W et al 2001 Acta Mater. 494005

[23] Kong L T, Li X Y, Lai W S, Liu J B and Liu B X 2002 Japan. J. Appl. Phys. 414503

[24] Gong H R, Kong L T, Lai W S and Liu B X 2003 Phys. Rev. B 68144201

[25] Zhang R F, Shen Y X, Gong H R, Kong L T and Liu B X 2004 J. Phys. Soc. Japan 732023

[26] Zhang R F, Kong L T, Gong H R and Liu B X 2004 J. Phys.: Condens. Matter 165251

[27] Zhang R F, Shen Y X, Yan H F and Liu B X 2005 J. Phys. Chem. B 1094391

[28] Dai X D, Li J H and Kong Y 2007 Phys. Rev. B 75052102

[29] Derlet P M, Nguyen-Manh D and Dudarev S L 2007 Phys. Rev. B 76054107

[30] Björkas C, Nordlund K and Dudarev S 2009 Nucl. Instrum. Meth. Phys. Res. B 2673204

Björkas C, Nordlund K and Dudarev S 2010 Nucl. Instrum. Meth. Phys. Res. B 2681529 (erratum)

[31] Wilson W D and Johnson R A 1972 Interatomic Potentials and Simulation of Lattice Defects ed P C Gehlen et al (New York: Plenum Press) p 375

[32] Henriksson K O E, Nordlund K, Keinonen J, Sundholm D and Patzschke M 2004 Phys. Scr. T108 95

[33] Juslin N, Erhart P, Träskelin P, Nord J, Henriksson K O E, Nordlund K, Salonen E and Albe K 2005 J. Appl. Phys. 98123520

[34] Li X-C, Shu X, Liu Y-N, Gao F and Lu G-H 2011 J. Nucl. Mater. 40812

[35] Samolyuk G D, Osetsky Y N and Stoller R E 2013 J. Phys.: Condens. Matter 25025403

[36] Heinola K, Ahlgren T, Nordlund K and Keinonen J 2010 Phys. Rev. B 82094102

[37] Daw M S and Baskes M I 1984 Phys. Rev. B 296443

[38] Bonny G and Pasianot R C 2010 Phil. Mag. Lett. 90559 